\title{
Estudo retrospectivo de crianças pré-termo no Ambulatório de Especialidades Jardim Peri-Peri
}

\author{
Retrospective study of preterm newborn infants at the ambulatory \\ of specialities Jardim Peri-Peri
}

\author{
Silvia Helena Tavares Lorena ${ }^{1}$ \\ José Martins Siqueira Brito ${ }^{2}$
}

\begin{tabular}{|c|}
\hline RESUMO \\
\hline 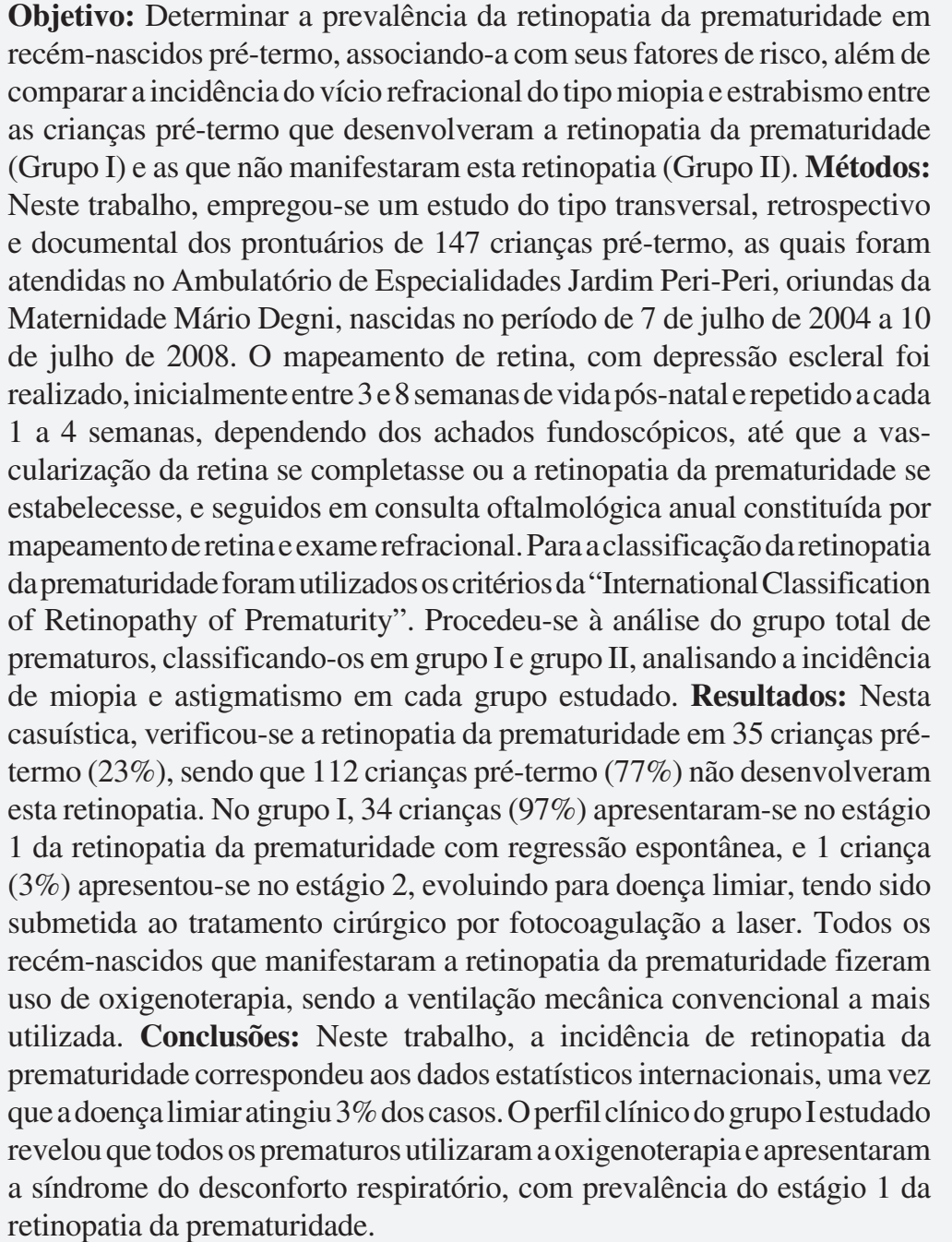 \\
\hline
\end{tabular}

Descritores: Retinopatia da prematuridade; Fatores de risco; Miopia/epidemiologia; Estrabismo; Prematuro

\section{INTRODUÇÃ̃O}

A prematuridade é definida como o nascimento abaixo de 37 semanas de gestação, segundo a Organização Mundial de Saúde - OMS (1961). Ela se 
divide em prematuridade moderada (32 semanas a 36 semanas de idade gestacional), prematuridade acentuada ( 28 semanas a 31 semanas de idade gestacional) e prematuridade extrema (abaixo de 28 semanas de idade gestacional).

A incidência de nascimentos prematuros relacionados aos países desenvolvidos é de 5 a $8 \%$, sendo que no Brasil é de $9 \%$.

A importância do estudo da prematuridade é devida à elevada incidência de morbiletalidade neonatal, além dos elevados custos das internações e ocorrência de sequelas de múltiplas naturezas, incluindo as de natureza oftalmológica, dentre as quais podemos citar: erros refrativos (miopia, astigmatismo), estrabismo, ambliopia e baixa visão.

A retinopatia da prematuridade é uma doença vasoproliferativa da retina, de etiologia multifatorial, ocorrendo em recém-nascidos pré-termo.

O recém-nascido prematuro apresenta uma retina imatura, parcialmente avascular, sendo a sua vascularização condicionada pela hipóxia local. Em alguns casos, pode ocorrer uma vascularização anômala, causando um processo fibrótico cicatricial e descolamento de retina; em outros casos, a vascularização anômala regride espontaneamente, não deixando sequelas ou levando a leves alterações fundoscópicas.

$\mathrm{O}$ aumento da pressão arterial de oxigênio após o nascimento $\left(\mathrm{PaO}_{2}\right.$ de $30 \mathrm{mmHg}$ para $80 \mathrm{mmHg}$ ), ocasiona uma constrição dos vasos da retina, que no prematuro ainda não estão completamente formados, levando à hipóxia da camada interna da retina, o que induz a síntese de uma proteína denominada VEGF (fator de crescimento do endotélio vascular), a qual, por sua vez, é responsável pelo crescimento de novos vasos sanguíneos ${ }^{(1)}$.

A fisiopatologia da retinopatia da prematuridade está relacionada ao fator de crescimento do endotélio vascular e a fatores não influenciados pela exposição ao oxigênio (fator de crescimento - insulina like-IGF-I). E, assim, quando esta proteína VEGF - está em níveis baixos, ocorre a inibição da formação dos vasos, e, quando a mesma está em níveis altos, estimula a neovascularização da retina.

Os principais fatores de risco para o desenvolvimento desta retinopatia são a prematuridade e o baixo peso ao nascer. Existem outros fatores de risco envolvidos e salientamos:

- flutuação nos níveis de $\mathrm{O}_{2}$ nas primeiras semanas de vida;

- ser a criança pequena para a idade gestacional - PIG;

- boletim de Apgar menor do que 7;

- fatores de risco maternos;

- fototerapia;

- transfusão sanguínea;

- persistência do canal arterial;

- síndrome do desconforto respiratório;

- sepse;

- fatores genéticos;

- asfixia perinatal;

- displasia broncopulmonar;

- hemorragia peri-intraventricular e

- IGF-I.
Os polimorfismos genéticos, podem alterar a função de genes que controlam a vascularização normal, o que explica a ocorrência da retinopatia da prematuridade em crianças com idade gestacional maior que 32 semanas e peso maior que $1500 \mathrm{~g}$ ao nascimento ${ }^{(2)}$.

Recentemente, o gene "Tubedow" foi clonado em laboratório e possui importante papel na regulação da neovascularização associada a esta retinopatia, constituindo um potencial terapêutico para o futuro ${ }^{(3)}$.

Cumpre assinalar que atualmente, está em estudo o uso de drogas antiangiogênicas para o tratamento de sequela de retinopatia da prematuridade grave $\mathrm{e}^{(4)}$.

\section{MÉTODOS}

Foram analisados retrospectivamente, os prontuários de 147 crianças prematuras, atendidas no Ambulatório de Especialidades Jardim Peri-Peri, oriundas da Maternidade Mário Degni, no período de 7 de julho de 2004 a 10 de julho de 2008.

As crianças pré-termo, no período em estudo, foram submetidas à avaliação oftalmológica (mapeamento de retina com depressão escleral), inicialmente entre 3 e 8 semanas, e seguidas em intervalos de 1 a 4 semanas, de acordo com os achados fundoscópicos, até que a vascularização da retina se completasse ou que a retinopatia da prematuridade se estabelecesse, e, após este procedimento, as crianças foram seguidas em consulta oftalmológica anual.

A oftalmoscopia binocular indireta foi realizada, após a dilatação das pupilas, utilizando-se colírios de tropicamida a $1 \%$ e fenilefrina a $2,5 \%$, uma gota em cada olho, com intervalo de 5 minutos, por 3 vezes, cerca de 40 minutos antes do exame. A oftalmoscopia binocular indireta foi realizada com lente de 20 dioptrias, utilizando-se um blefarostato, com a intenção de manter as pálpebras das crianças abertas, instilando-se, anteriormente, gotas de colírio anestésico. Empregou-se, ainda, um gancho de estrabismo como depressor escleral.

As crianças prematuras foram submetidas à refração sob cicloplegia, com retinoscópio e régua de esquiascopia, com 4 meses de idade e acompanhados, subsequentemente, por períodos anuais.

Para a classificação da retinopatia da prematuridade, foram utilizados os critérios da "International Classification of Retinopathy of Prematurity".

Os fatores de risco estudados para esta retinopatia foram: oxigenoterapia, síndrome do desconforto respiratório, baixo peso $(<1500 \mathrm{~g})$, fototerapia, boletim de Apgar $<7$, sepse, transfusão sanguínea, hemorragia intraventricular e gestação múltipla. Os prontuários dos prematuros foram fonte de avaliação dos fatores de risco para a retinopatia da prematuridade a que foram submetidas.

As crianças prematuras deste trabalho foram avaliadas quanto à prevalência de vício refracional e estrabismo aos 4 meses de vida e anualmente até completarem 4 anos. 
Considerou-se cada olho como unidade amostral e as associações dos fatores de risco, incidência de miopia e estrabismo, foram analisadas pelo teste do Qui-quadrado. Adotouse como nível de significância, o valor de 0,05 (5\%) para todos os testes. Níveis descritivos (P), inferiores a esse, foram considerados significantes.

\section{RESULTADOS}

Da população de 147 crianças examinadas, 112 (76\%) não desenvolveram a retinopatia da prematuridade (Grupo II) e 35 (23\%) desenvolveram esta retinopatia (Grupo 1). O gráfico 1 simplifica esta amostragem.

Neste trabalho, verificou-se que dentre os recém-nascidos pertencentes ao grupo I, 34 prematuros (97\%) tiveram a regressão espontânea da retinopatia da prematuridade e apenas 1 prematuro $(3 \%)$ evoluiu para doença limiar, necessitando de tratamento (fotocoagulação a laser), 26 prematuros $(74 \%)$ apresentaram idade gestacional igual ou abaixo de 32 semanas (média de 30 semanas), 25 prematuros $(71,4 \%)$ apresentaram peso ao nascimento igual ou abaixo de $1500 \mathrm{~g}$ (média de $1104 \mathrm{~g}), 18$ prematuros $(51,4 \%)$ eram do sexo feminino e 17 prematuros $(48,5 \%)$ eram do sexo masculino.

É importante ressaltar que, segundo a literatura, a retinopatia da prematuridade incide igualmente em ambos os sexos, o que é confirmado neste trabalho.

No estudo dos fatores de risco para a retinopatia da prematuridade, além de salientar estes dois principais: a imaturi- dade retiniana e a oxigenoterapia, destacamos outros fatores de risco:

- Fototerapia: 22 crianças (62\%) foram submetidas a este procedimento;

- Gestação múltipla: 2 crianças $(5,7 \%)$ foram fruto de gestação múltipla;

- Transfusão sanguínea: 16 crianças $(45,7 \%)$ submeteramse a este procedimento;

- Apgar menor que 7: 19 crianças (54\%) obtiveram este valor;

- Hemorragia intraventricular: 6 crianças (17\%) tiveram este fator de risco;

- Sepse: 16 crianças $(45,7 \%)$ apresentaram esta patologia;

- Síndrome do desconforto respiratório: 35 crianças (100\%) tiveram esta patologia.

Todos os prematuros fizeram uso de oxigenoterapia, sendo a ventilação mecânica convencional a mais utilizada. Em relação ao tempo de oxigenoterapia: 13 crianças $(37,14 \%$ dos casos) foram expostas por tempo menor que 1 semana, e 16 crianças $(45,71 \%$ dos casos) permaneceram até 20 dias em oxigenoterapia, e 6 crianças $(17,14 \%)$ por mais de 20 dias.

Os achados estatisticamente significantes $(p<0,05)$, neste estudo foram: uso de oxigenoterapia, recém-nascido com idade gestacional menor ou igual a 32 semanas, fototerapia, boletim de Apgar menor que 7, sepse, recém-nascido com peso menor ou igual a $1500 \mathrm{~g}$ e síndrome do desconforto respiratório.

O gráfico 2 exemplifica a análise destes fatores de risco para esta retinopatia, conforme mencionado acima.

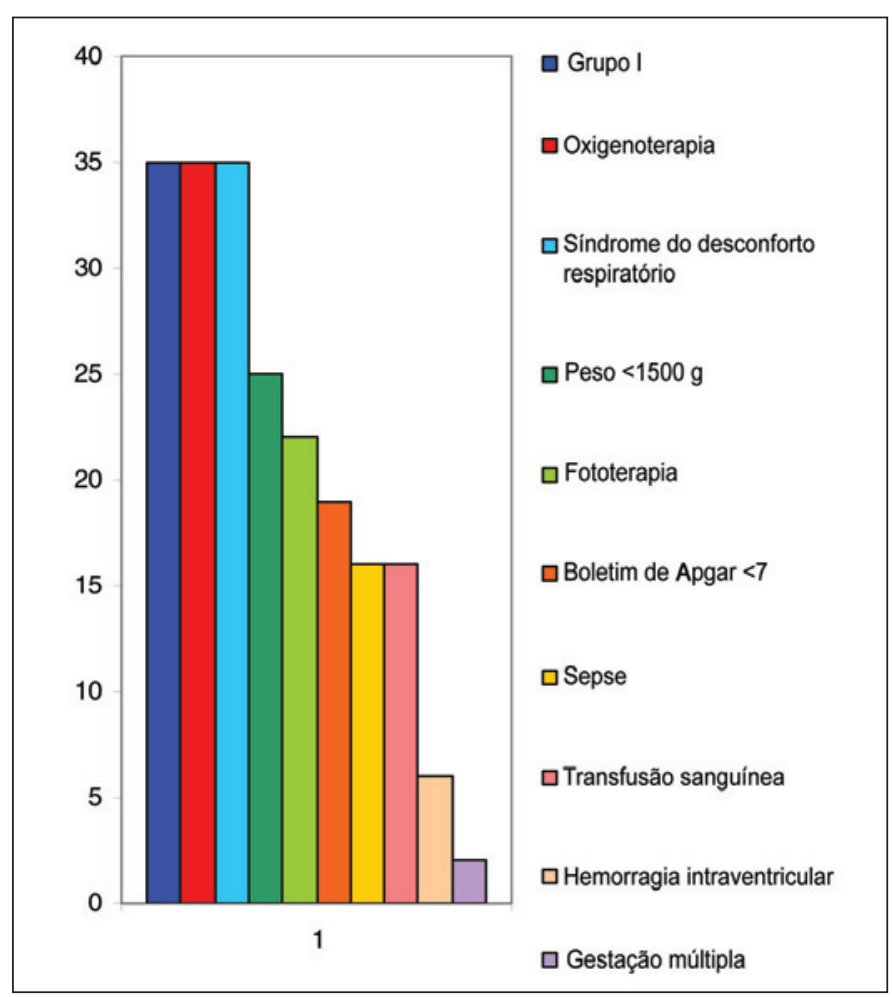

Gráfico 2 - Fatores relacionados à retinopatia da prematuridade

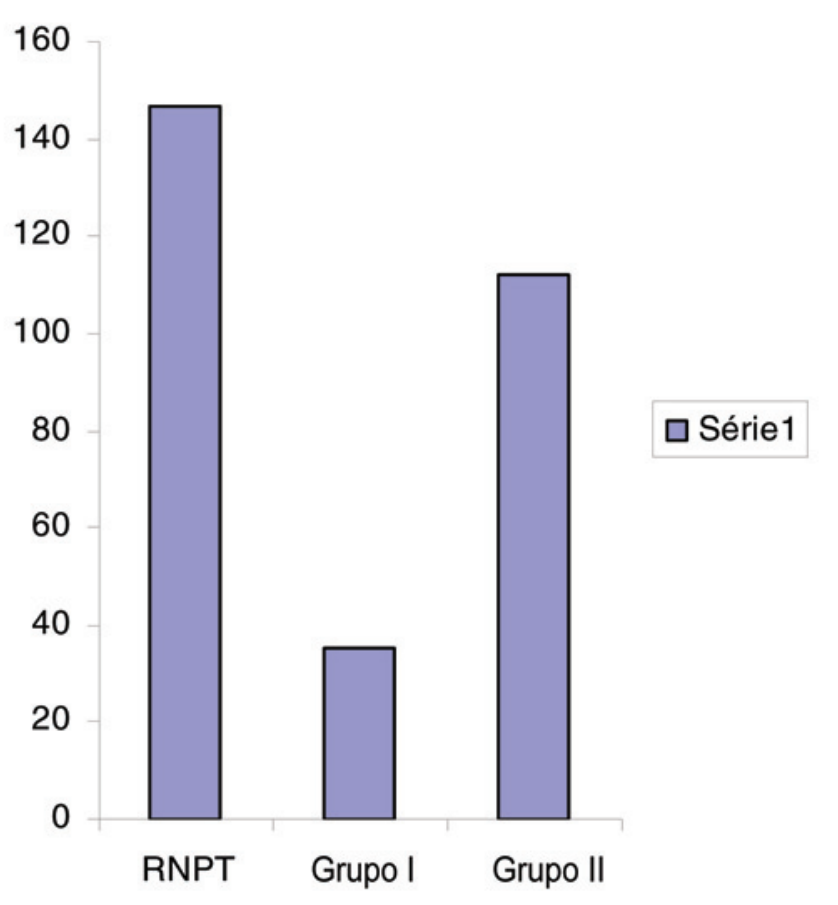

ráfico 1 - Características da população estudada 
Neste trabalho, a incidência do vício refracional do tipo miopia, entre os grupos estudados, teve a seguinte distribuição: no grupo I, 20 crianças (58\%) tiveram este vício refracional e, no grupo II, 71 crianças $(63,3 \%)$.

A incidência de estrabismo foi de 5 crianças (14\%), no grupo I, e de 8 crianças $(7,1 \%)$, no grupo II, o que confirma os dados da literatura, a qual relata uma maior incidência de miopia e de estrabismo em crianças prematuras.

Neste trabalho os achados relativos a erro refracional tipo miopia e estrabismo, nos grupos estudados, foram estatisticamente significantes (adotando-se $\mathrm{p}<0,05$ ).

O gráfico 3 exemplifica o que foi exposto acima.

\section{DISCUSS ÃO}

As crianças prematuras apresentaram uma maior incidência de miopia e estrabismo convergente, o que foi confirmado na literatura mundial ${ }^{(5)}$. Neste trabalho, verificou-se uma prevalência de $71,4 \%$ da retinopatia da prematuridade em prematuros com peso inferior a $1500 \mathrm{~g} \mathrm{e} \mathrm{em} 74 \%$ com idade gestacional abaixo de 32 semanas, o que é também confirmado na literatura ${ }^{(6)}$.

O risco desta retinopatia aumenta proporcionalmente quanto mais precoce a idade gestacional e menor o peso ao nascimento, e maior taxa de $\mathrm{O}_{2}$ suplementar entregada, de acordo com a literatura ${ }^{(7)}$. Neste trabalho, salientamos os fatores de risco associados a esta retinopatia e descreveremos cada um deles a seguir.
A síndrome do desconforto respiratório foi frequente no grupo I, porém está relacionada ao grau de prematuridade, o que confirma a hipótese que esta síndrome é sinal de imaturidade e não fator de risco para a retinopatia da prematuridade.

A administração de surfactante exógeno foi maior no grupo I, devido à maior imaturidade pulmonar, porém, segundo alguns autores, não constatou interferência do surfactante exógeno na incidência da retinopatia da prematuridade ${ }^{(8)}$.

Existem controvérsias na associação entre a retinopatia da prematuridade e transfusões, uma vez que, as transfusões são realizadas em crianças debilitadas, necessitando de oxigenoterapia prolongada ${ }^{(9)}$.

O retardo de crescimento intraútero e/ou baixo peso e a sepse se associaram ao risco da retinopatia da prematuridade ${ }^{(10)}$.

A asfixia perinatal não representa um fator de risco para esta retinopatia, uma vez que está relacionada à prematuridade ${ }^{(11)}$.

A persistência do canal arterial, causando hipoperfusão da retina, representa um fator de risco para esta retinopatia, porém outros autores afirmam que não é representativa ${ }^{(12-13)}$.

A displasia broncopulmonar e a hemorragia peri-intraventricular estão relacionados a esta retinopatia, porém há controvérsias, uma vez que estas complicações são comuns em prematuros clinicamente instáveis ${ }^{(14-15)}$.

A deficiência de IGF-I em prematuros está relacionada com a incidência da retinopatia da prematuridade ${ }^{(16-18)}$.

É referido na literatura que 1/3 dos casos desta retinopatia tem origem em condições pré-natais, como a doença hipertensiva da gestação ${ }^{(19)}$.

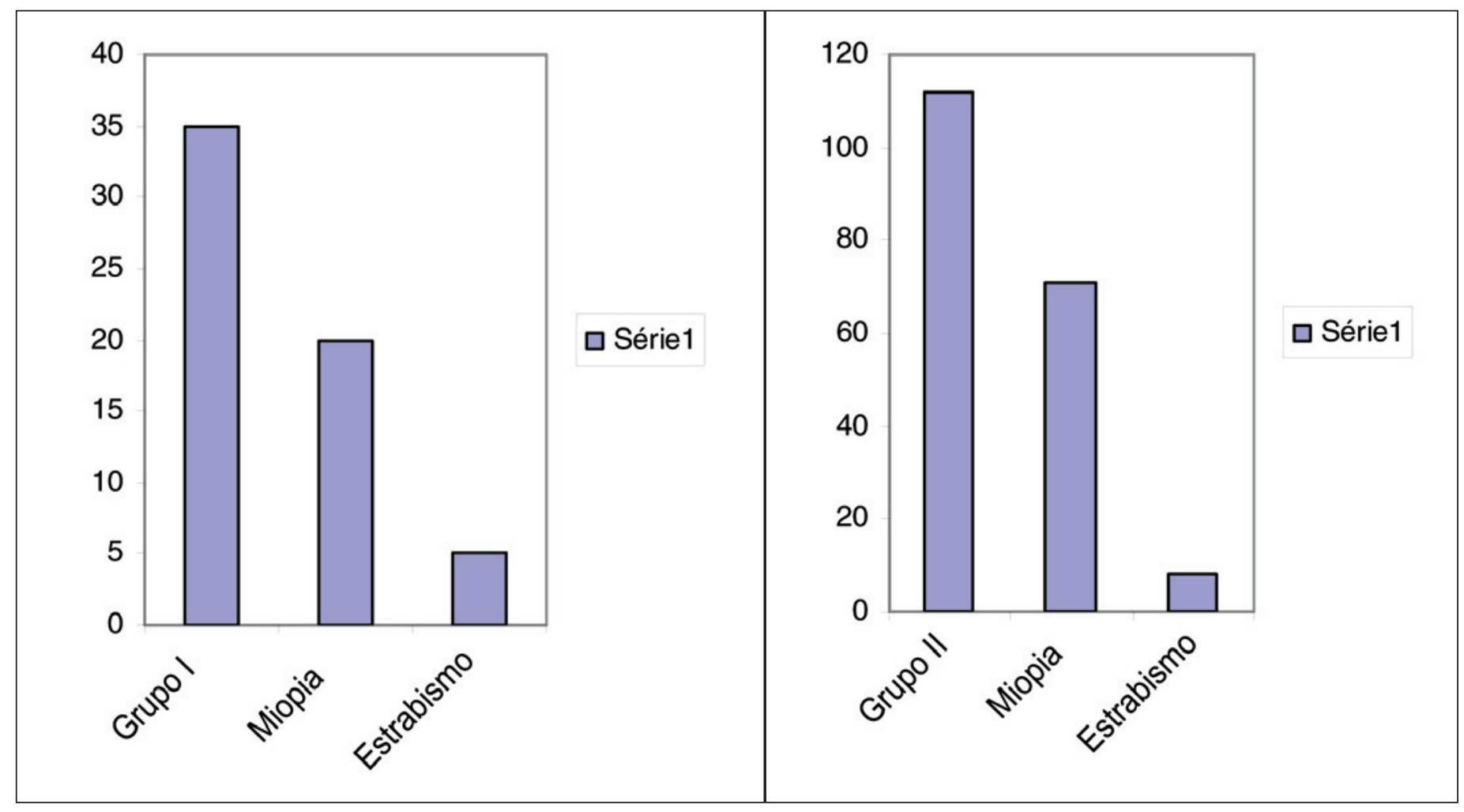

Gráfico 3 - Incidência de miopia e estrabismo nos grupos I e II 


\section{CONCLUSÕES}

Neste trabalho, a incidência da retinopatia da prematuridade, correspondeu aos dados estatísticos internacionais, uma vez que, a doença limiar atingiu $3 \%$ dos casos.

O perfil clínico do grupo I estudado revelou que todos os prematuros utilizaram a oxigenoterapia e apresentaram a síndrome do desconforto respiratório, com prevalência do estágio 1 da retinopatia da prematuridade.

A prevenção desta retinopatia consiste na diminuição do nível de oxigenação para o prematuro, medidas para prevenir o parto prematuro e a indução pré-natal da maturidade pulmonar pela corticoterapia.

O desenvolvimento da retinopatia da prematuridade é inversamente proporcional ao peso e a idade gestacional ao nascimento.

\section{ABSTR ACT}

Purpose: To evaluate the prevalence of retinopathy of prematurity preterm newborn infants, associating it with its risk factors besides comparing the incidence of refractive errors like myopia and strabismus among preterm children who developed retinopathy of prematurity (Group I) and those who did not present this retinopathy (Group II). Methods: A cross-sectional, retrospective and documental study of records of 147 preterm children who were examined at the "Ambulatório de especialidades Jardim Peri Peri" (Ambulatory of Specialties Jardim Peri Peri) was used in this paper. These children were born between July $7^{\text {th }}, 2004$ and July $10^{\text {th }}, 2008$ at "Maternidade Mário Degni” (Maternity Mário Degni).Fundus examination with escleral depression was first carried out between the $3^{\text {rd }}$ and $8^{\text {th }}$ weeks after birth and repeated every 1 to 4 weeks depending on the retinal imaging findings, until retina vascularization was complete or retinopathy of prematurity was present and it was followed by an annual ophthalmologic followup comprised of fundus examination and refraction. International Classification of Retinopathy of Prematurity criteria was used to classify the retinopathy of prematurity. Analysis of the whole group of preterm newborns was made and classified in group I and group II, and the incidence of Myopia and Astigmatism in each group was analyzed. Results: This casuistry showed that retinopathy of prematurity was present in 35 preterm children (23\%) and 112 preterm children (77\%) did not develop this retinopathy. In Group I, 34 children (97\%) were classified as stage I Retinopathy and had spontaneous regression and 1 child (3\%) was in stage II Retinopathy and developed a threshold disease. Conclusions: In this study, the incidence of retinopathy of prematurity was similar to the international statistic data, since the threshold disease reached
$3 \%$ of the cases. The clinical profile of group I showed that all the preterm newborn infants used oxygen support and presented Respiratory Distress syndrome, with the prevalence of stage I.

Keywords: Retinopathy of prematurity; Risk factors; Myopia/ epidemiology; Strabismus; Infant, premature

\section{REFERÊNCIAS}

1. Fielder AR, Shaw DE, Robinson J, Ng YK. Natural history of retinopathy of prematurity: a prospective study. Eye. 1992;6(Pt 3):233-42.

2. The natural ocular outcome of premature birth and retinopathy; Status at 1 year. Cryotherapy for Retinopathy of Prematurity Cooperative Group. Arch Ophthalmol. 1994;112(7):903-12. Comment in: Arch Ophthalmol. 1995;113(7):850-1. Arch Ophthalmol. 1995;113(6):697-8.

3. Quinn GE, Dobson V, Saigal S, Phelps DL, Hardy RJ, Tung B, Summers CG, Palmer EA, CRYO-ROOP Cooperative Group. Health-related quality of life at age 10 years in very low birth-weight children with and without threshold retinopath of prematurity. Arch Ophthalmol. 2004;122(12):1659-66.

4. Heath, P. Pathology of retinopathy of prematurity: retrolental fibroplasia. Am J Ophthalmol. 1951;34(9):1249-59.

5. Phelps DL. Vision loss due to retinopathy of prematurity. Lancet. 1981;1(8220 Pt 1):p.606.

6. Palmer EA, Flynn JT, Hardy RJ, Phelps DL, Phillips CL, Schaffer DB, et al. Incidence and early course of retinopathy of prematurity. The Cryotherapy for Retinopathy of Prematurity Cooperative Group. Ophthalmology. 1991;98(11): 1628-40.

7. Graziano RM, Leone CR. [Frequent ophthalmologic problems and visua development of preterm newborn infants]. J Pediatr (Rio J). 2005;81(1Suppl): S95-100. Portuguese.

8. Graziano RM, Leone CR, Cunha SL, Pinheiro AC. [Prevalence of retinopathy of prematurity in very low birth weight infants]. J Pediatr (Rio J);1997;73(6): 377-82. Comment in: J Pediatr (Rio J); 1997;73(6):361-2. Portuguese.

9. Graziano RM. Retinopatia da prematuridade: contribuição ao estudo da ocorrência e análise dos fatores de risco [tese]. São Paulo: Faculdade de Medicina da Universidade de São Paulo; 1994.

10. Avery GB, Glass P. Retinopathy of prematurity: what causes it? Clin Perinatol. 1988;15(4):917-28.

11. Pierce A, Foley ED, Smith LE. Regulation of vascular endothelial growth factor by oxygen in a model of retinopathy of prematurity. Arch Ophthalmol. 1996; 114(10):1219-28. Erratum in: Arch Ophthalmol. 1997;115(3):427. Comment in: Arch Ophthalmol. 1996;114(10):1252-4; Arch Ophthalmol. 2000;118(8):1121-2.

12. Park KH, Hwang JM, Choi MY, Yu YS, Chung H. Retinal detachment of regressed retinopathy of prematurity in children aged 2 to 15 years. Retina. 2004;24(3):368-75.

13. Holmström M, el Azazi M, Kugelberg U. Ophthalmological long-term follow up of preterm infants: a population based, prospective study of refraction and its developments. Br J Ophthalmol. 1998;82(11):1265-71.

14. Charles JB, Ganhier R Jr, Appiah AP. Incidence and characteristics of the retinopathy of prematurity in a low-income inner-city population. Ophthalmology. 1991;98(1):14-7.

15. The International Committee for the Classification of Retinopathy of Prematurity. The International Classification of Retinopathy of Prematurity Revisited. Arch Ophthalmol. 2005;123(7):991-9. Comment in: Arch Ophthalmol. 2006; 124(11):1669-70.

16. Campochiaro PA, Molecular targets for retinal vascular diseases. J Cell Physiol. 2007;210(3):575-81

17. Eichler W, Yafai Y, Wiedermann P, Fengler D. Antineovascular agents in the treatment of eye diseases. Curr Pharm Des. 2006;12(21):2645-60.

18. Anlar B, Sullivan KA, Feldman EL. Insulin-like growth factor-I and central nervous system development. Horm Metab Res. 1999;31(2-3):120-5.

19. Good WV, Gendron RL. Retinopathy of prematury. Ophtalmol Clin North Am. 2001;14(3):513-9. 